\title{
La dimension éthique et politique de la révélation prophétique chez les falāsifa
}

\author{
Meryem Sebti
}

\section{Introduction}

L'héritage grec a nourri et influencé en profondeur une tradition philosophique en langue arabe'. Les œuvres éthiques et politiques d'Aristote, dont l'Éthique à Nicomaque - qui aura une influence déterminante sur la pensée éthique et politique des philosophes musulmans -, sont traduites ; des extraits de la République et les Lois de Platon deviennent accessibles en arabe.

Il est dorénavant d'usage de qualifier de «classique » cette période, qui va du IX ${ }^{\text {ème }}$ au XII ${ }^{\text {ème }}$ siècle. C'est durant cette époque que cet héritage grec est réinterprété par les philosophes musulmans. Pour notre propos, nous nous limiterons à cette période classique et aux philosophes qui ont revendiqué leur lien explicite avec l'héritage grec et notamment avec Aristote. Ils sont nommés falāsifa ${ }^{2}$. Dans le monde musulman, cette science a été tôt caractérisée comme étant allogène ${ }^{3}$, attestant par leur nom même le lien avec le monde grec. Il n'en reste pas moins que le traitement par les falāsifa de la question de la prophétie aura une influence déterminante sur certains théologiens ash'arites, et que la synthèse avicennienne constitue une étape majeure dans la constitution de la prophétologie islamique, de telle sorte qu'on peut considérer qu'il y a un avant et un après Avicenne, pour ce qui est de la doctrine de la prophétie dans le monde musulman 4 .

A moins de simplifications hâtives, il n'est pas possible d'esquisser les contours d'une prophétologie qui serait commune à tous les falāsifa. Al-Kindī

1 Pour l'histoire du mouvement de traduction gréco-arabe, cf. Gutas, Greek Thought, Arabic Culture. Pour la transmission des œuvres de Platon en arabe, voir l'article de Gutas, "Platon. Tradition arabe".

2 Shahrastānī (m. 1158), Kitāb al-milal wa-l-niḥal, III, 501-636. Cf. Shahrastānī, Le livre des religions et des sectes, $363-67$.

3 Peter Adamson, indique que c'est ainsi qu'ils sont caractérisés par al-Khwārizmī dans son Mafātị̂ al-úūm (écrit autour de 977), Adamson, Al-Kindi, 21.

4 Ayman Shihadeh montre comment la doctrine de la prophétie avicennienne a influencé celle de Rāzī, The Theological Ethics ; voir aussi Griffel, Al-Ghazālì’s Philosophical Theology. 
(m. après 870), Abū Bakr al-Rāzī (864-925) al-Fārābī (m. 950), Avicenne (9801037), Ibn Bājja (m. autour de 1138), Ibn Ṭufayl (1110-1185) et Averroès (1126-1198) ont néanmoins, en dépit de leurs différences et de leurs différents, tenté de rendre compte rationnellement du phénomène de la prophétie ; l'intégrant pour certains d'entre eux - dans une cosmologie complexe de type émanatiste. La position d'Abū Bakr Rāzī sur la prophétie n'est connue qu'au travers de l'œuvre de son adversaire, l'ismaélien Abū Ḥātim al-Rāzī qui la rapporte dans son Kitāb a lām al-nubuwwwa ${ }^{5}$. En règle générale, ce qui intéresse les falāsifa ce n'est pas tant le personnage historique ou sacré du prophète Muhammed qu'une analyse de ce que signifie d'un point de vue philosophique la présence parmi les hommes d'un homme porteur d'une Loi dont l'origine est divine. Leur approche de la prophétie demeure généralement dans la lignée de la tradition philosophique grecque, dans la mesure où ils cherchent à rendre compte rationnellement de l'expérience prophétique. Les principes de leur épistémologie permettent d'expliquer les visions prophétiques et le mode de transmission de la révélation (al-wahy). Cependant, alors que Fārābī demeure dans une description générique de la prophétie, Avicenne, notamment dans le livre x de la Métaphysique du Shifä), identifie clairement le prophète avec le prophète de l'islam par la médiation duquel le Coran a été révélé aux hommes.

\section{$2 \quad$ Raison et foi}

Ce qui caractérise la falsafa, c'est la conviction inébranlable que la raison humaine peut accéder par ses propres moyens au fondement ontologique et métaphysique de la réalité. L'acte créateur de Dieu, Sa providence, l'unicité absolue de Son essence : toutes ces questions sont du ressort de notre humaine raison. Dès lors, se pose de façon aigue la question de savoir pourquoi donc la prophétie et le message qu'elle révèle aux hommes sont nécessaires alors même qu'une certaine catégorie d'hommes - les philosophes - ont accès, par la ressource de leur seule raison, à la vérité ultime. On est en droit de se demander si la prophétologie n'est pas pour les philosophes un exercice de style obligé, destiné à satisfaire un auditoire musulman. Bien que légitime, cette question reçoit une réponse négative. Tous affirment la nécessité de la prophétie, même

5 Rāzī, Kitāb a lām al-nubuwwwa. Contrairement à l'idée longtemps soutenue sur la base de l'ouvrage de son adversaire selon laquelle Abū Bakr Rāzī rejetait la prophétie, Marwan Rashed montre de façon convaincante que Rāzī accorde au prophète une fonction éthique d'éducation des âmes, cf. Rashed, "Abū Bakr Rāzī” 169-82. 
si les arguments qui fondent cette nécessité sont conçus différemment par les uns et les autres.

Leur conviction commune est que la philosophie, qui exprime mieux que toute autre science la puissance de la raison, n'est pas muette face aux données de la révélation. Elle n'est pas là certes pour s'y substituer, mais elle peut les fonder rationnellement et même leur apporter un poids supplémentaire, notamment lorsqu'il s'agit de dogmes sujets à controverse avec d'autres religions. La philosophie vient renforcer la révélation. Elle vient attester par le pouvoir de la raison sa véracité. Ainsi, pour al-Fārābī et Avicenne, la particularité du prophète doit être rationnellement fondée ; elle s'inscrit dans une métaphysique et une cosmologie qui permettent de rendre raison des capacités singulières du prophète, notamment noétique.

Al-Kindī dans son ouvrage Risāla fì kammiyyat kutub Arisțūțālìs ${ }^{6}$ distinguait déjà la connaissance du prophète de celle des autres hommes. Celle du prophète vient directement de Dieu par révélation; elle ne s'acquiert pas dans le temps alors que les hommes doivent entreprendre un long apprentissage pour atteindre la connaissance philosophique. Cependant, bien qu'al-Kindī distingue le mode d'accès à la connaissance du prophète de celui des hommes ordinaires, il ne dit pas que la connaissance à laquelle parviennent les prophètes est différente de celle à laquelle accèdent les philosophes. Les philosophes l'acquièrent par l'effort et l'étude et le prophète instantanément par révélation. Cette doctrine aura une influence déterminante sur les doctrines noétiques des falāsifa qui viennent après lui. L'intellect étant la plus noble des facultés humaines, celle qui le distingue des animaux et le rend semblable aux êtres célestes, il va de soi que le prophète, qui est le plus noble des hommes, doit avoir un intellect parfaitement accompli. Al-Fārābī et Avicenne lui attribuent aussi une imagination singulièrement développée, qui lui permet de transcrire les intelligibles reçus de l'intellect agent en symboles, accessibles à ceux dont la capacité intellectuelle ne leur permet pas d'avoir accès aux démonstrations apodictiques de la métaphysique ${ }^{7}$. Averroès lui accorde, en sus d'un intellect

6 Rasāil al-Kindī al-falsafiyya, 373; traduction anglaise dans Adamson and Pormann, The Philosophical Works of al-Kindī.

7 Il existe néanmoins une différence importante entre la doctrine de l'imagination prophétique de Fārābī et celle d'Avicenne. En raison des particularités de sa cosmologie, Avicenne établit une connexion entre les images contenues dans les âmes des sphères célestes, qui sont dotées d'imagination, et l'imagination des hommes et en particulier avec l'imagination du prophète, qui est pleinement réceptive à cette donation. Fārābī considère quant à lui que l'intellect agent émane des formes intellectuelles sur l'intellect et que l'imagination les transforme en images. Il récuse l'existence d'âmes des sphères. Cette distinction entre les doctrines des deux philosophes a une conséquence importante sur le statut des images reçues par l'imagination du prophète. 
pleinement réalisé, la maîtrise de l'outil rhétorique qui lui permet de s'adresser au peuple par métaphore et d'être aisément compris de lui. Selon lui, le prophète donne à méditer aux hommes du commun (al-āmma) des symboles qui permettent à tout un chacun d'avoir une représentation imagée de la vérité ultime sans être déconcerté par lâpre rigueur de la vérité métaphysique ${ }^{8}$. Le prophète apparait dès lors comme un médiateur nécessaire sans lequel la vie en commun basculerait dans le chaos. Grâce à la sharîa en effet, la Loi divine dont il est le transmetteur, les hommes du commun peuvent mener une vie vertueuse. Quant aux autres, lélite des hommes (al-khāșșa) à laquelle appartiennent les philosophes, ils se doivent de chercher à connaitre le fondement de la réalité à l'aide de la méthode démonstrative : selon Averroès, il s'agit là pour eux d'une obligation légale. C'est néanmoins la Loi révélée qui sert de ciment social et permet la vie en commun: elle assure la constitution et la pérennité de la umma (la communauté religieuse). Dans son Faṣl al-Maqāl, Averroès insiste sur le fait que les versets autour desquels il y a consensus dans la communauté musulmane ne peuvent être discutés par les philosophes ${ }^{9}$.

\section{$3 \quad$ Éthique et politique}

L'homme est un animal social qui ne peut survivre hors d'une communauté politique $^{10}$. Or, la cité musulmane est régie par la Loi révélée, la sharīa $a$ qui est transmise aux hommes par le prophète. Ainsi que le montre Muhsin Mahdiil ${ }^{11}$ la

8 "Les choses qui, en raison de leur abscondité, ne peuvent être connues que par la démonstration, Dieu a fait à Ses serviteurs qui n'ont pas accès à la démonstration, à cause de leurs dispositions innées, ou de leurs habitudes, ou à défaut des conditions [qui leur eussent permis] cet apprentissage, la grâce de leur en présenter des symboles et des allégories, et de les convier à accorder leur assentiment à ces symboles, car à ceux-ci il est possible d'assentir au moyen des arguments qui sont communs à tous, c'est-à-dire les dialectiques et les rhétoriques", Averroès, Traité décisif, 140 (arabe); 141 (français).

9 “Notre propos a fait apparaître qu'il y a dans la Révélation des énoncés auxquels il faut attribuer leur sens obvie et qu'il n'est pas permis d'interpréter, et dont l'interprétation est infidélité si elle met en cause des principes [dogmatiques] fondamentaux; ou innovation blâmable si elle met en cause quelque chose en deçà de ces principes", Traité décisif, 140142 (arabe); 141-43 (français).

10 Aristote définissait l'homme ainsi: "L'homme est par nature un animal politique", Politique, 1253 a 2-3, La Politique d'Aristote n'a pas été traduite en arabe, néanmoins cette définition de l'homme comme "animal politique" a été connue des falāsifa. Sur la question de la traduction de la Politique en arabe, cf. Brague, "Note sur la traduction arabe de la Politique", 423-33. S. Vasileos affirme que quelques fragments de la Politique auraient existé en arabe, cf. "A Note on the Transmission of Aristotle".

Muhsin Mahdi, "The Political Orientation". 
sharîa enjoint aux hommes de se considérer comme étant, bien que créatures privilégiées auxquelles Dieu d'adresse par l'intermédiaire de son prophète, les éléments d'un ensemble qui les inclut et les dépasse. Répondre aux réquisits de la sharīa nécessite une attitude vertueuse de la part de l'homme. Il lui incombe de mettre de côté les exigences de son individualité pour permettre la bonne marche de la umma. C'est là, comme le remarque Muhsin Mahdi, une exigence commune avec la démarche philosophique, qui requiert de l'homme une attitude vertueuse afin de pouvoir satisfaire à la rigueur de l'exercice de la philosophie, lequel ne peut être accompli par un homme que mènent ses passions. Ainsi la cité religieuse et la cité philosophique sont toutes deux des cités vertueuses ${ }^{12}$. Al-Fārābī a été le premier à concevoir une interconnexion étroite entre le travail éthique sur soi et la dimension proprement politique de la vie humaine. Cette doctrine, qui est au cœur de sa pensée, aura une influence décisive sur ses successeurs, en particulier sur Avicenne et Averroès. Ainsi, al-Fārābī affirme dans Kitāb Taḥṣll al-sa'āda que "philosophe », "princeps », «roi », «nomothète» et «imam» ont la même signification ${ }^{13}$. Dans la philosophie grecque comme dans le monde musulman, une place centrale est accordée à la loi, et c'est cet idéal commun qui permet à Fārābī de réaliser une synthèse entre la source grecque et islamique.

La lecture des œuvres politiques et éthiques grecques a conduit Fārābī et ses épigones à saisir plus complètement le caractère politique de la sharî́a en islam $^{14}$. Pour ces philosophes, comme le montre Muhsin Mahdi, la révélation n'est pas seulement la communication directe entre Dieu et l'homme; une transmission de croyances, un dialogue entre un Dieu personnel de justice et d'amour à un homme qu'il a créé à son image ; c'est surtout et avant tout une Loi qui engage l'homme qui doit vivre dans une société politiquement organisée afin de pouvoir réaliser pleinement sa destinée. Cette Loi est conçue par les falāsifa comme étant celle de la cité idéale. Elle englobe tous les détails de la vie, de la codification des mariages et des divorces à la préparation de la vie éternelle. Elle permet à l'homme de bien vivre ici-bas et de préparer sa vie dans l'au-delà. Ce double aspect de la sharîa est ce qui permet de la caractériser

\footnotetext{
12 Muhsin Mahdi, "The Political Orientation" 5.

13 “Ainsi, 'imam,' 'philosophe' et 'nomothète' (wäḍi' al-nawāmìs) ont la même signification", Kitāb Tahṣill al-sa'āda, 92.

14 Dimitri Gutas, considère, notamment contre Muhsin Mahdi et beaucoup d'autres, que la dimension politique de l'œuvre de Fārābī a été exagérée et qu'elle mériterait d'être revue, cf. Gutas, "The meaning of madanī". Pour la transmission des œuvres politiques d'Aristote en arabe, on peut consulter, Janssens, "Ibn Bājja and Aristote". Dans cet article, Jules Janssens se réfère à l'Éthique à Nicomaque, à la Rhétorique et à l'Histoire des Animaux d'Aristote et étudie l'influence de ces ouvrages sur la pensée politique d'Ibn Bājja.
} 
comme Loi idéale. Aucune autre loi conçue par un nomothète humain ne pourrait réunir ces deux aspects pourtant indissociables de la vie humaine. La sharĩa permet de fonder la cité idéale, comme la République était pour Platon l'état idéal.

La sharīa, par l'intermédiaire du prophète, offre les lois de bonnes conduites; les lois qui permettent à l'homme d'être vertueux. Tous les falāsifa n'ont pas jugé nécessaire que l'homme vive dans une cité vertueuse pour atteindre la perfection intellectuelle. Ibn Țufayl, dans son Hayy Ibn Yaqzān, rompt avec la doctrine élaborée par al-Fārābī selon laquelle le philosophe doit penser les conditions de la cité vertueuse qui permet à tous de mener une vie vertueuse et au philosophe de réaliser la pleine perfection de son intellect. Même si Avicenne et Averroès avaient réinterprété cette doctrine, chacun différemment, elle n'en restait pas moins un horizon doctrinal à partir duquel ils orientaient leur propre doctrine. Pour Ibn Țufayl, au contraire, le philosophe doit cheminer dans la solitude afin d'atteindre sa perfection.

Pour les falāsifa, l'éthique et le politique sont indissociables (à l'exception peut-être d'Ibn Ṭufayl). Cette doctrine a une forte incidence sur leur prophétologie, puisque le prophète est celui qui transmet la loi morale, loi sans laquelle la vie commune - la vie politique donc - n'est pas possible. Le prophète est donc le garant de la vie politique. Ce sont les lois qu'il a reçues par révélation qui cimentent la vie commune et organisent la cité.

Cette interconnexion entre l'éthique et le politique trouve sa source dans la pensée de Platon ainsi que dans la conception proprement musulmane de la sharîa. Il existe néanmoins des nuances importantes entre les différents falāsifa. Au cours de cet article, je vais exposer brièvement la conception de l'éthique d'Avicenne puis celle d'Averroès pour montrer comment, malgré un socle doctrinal commun, chacun de ces deux philosophes conçoit différemment la fonction éthique et politique du prophète.

Éthique et prophétie selon Avicenne : une esquisse

C'est dans un exposé consacré à la prophétie dans la Métaphysique du Shifä' qu'Avicenne cherche à fonder sa nécessité. Ce chapitre qui constitue le deuxième chapitre du livre x de la Métaphysique est intitulé « Fĩ ithbāt al-nubuwwa wa-kayfiyyat da'wat al-nabī ilā Allah ta'âlā, wa-l-ma'ād ilayhi $\gg^{15}$ (De la preuve de la prophétie et de la manière dont le prophète appelle à Dieu qu'Il soit exalté, et du retour à Lui). Suivons le philosophe dans son argumentation : l'homme,

15 Shifä': Ilāhiyyāt, 441-43. 
note-t-il - dans la droite lignée de Platon ${ }^{16}$ - se distingue des autres animaux par le besoin (al-hāja) qui est le sien de vivre en société. L'homme ne peut vivre hors de la société, car c'est dans cette structure organisée que chacun fournit à l'autre ce dont il a besoin pour sa survie. Ainsi, les hommes doivent nécessairement vivre en association (mushāraka), or toute association doit reposer sur un système de transactions réciproques (wa-lā tatimmu al-mushāraka illā bi-l-mu'āmala). Les transactions réciproques ne peuvent se réaliser que si la loi et l'équité prédominent (wa-lā budda fí-l-mu'āmala ${ }^{17}$ min sunna wa 'adl), or la loi et l'équité requièrent un législateur et un dispensateur de justice qui les définisse et les applique. Ce législateur doit pouvoir s'adresser aux hommes afin de les contraindre à adhérer à la loi. Il doit donc être un homme (wa-lā budda min an yakūna hädhā insānan). Il ne peut pas laisser les hommes à leurs opinions concernant la loi, car alors ils divergeraient, chacun considérant être dans son bon droit. Ainsi, pour la survie de l'espèce humaine - qui ne peut s'accomplir qu'en société - l'existence d'un tel homme est absolument nécessaire. Il est impossible que la Providence divine qui nous a octroyé tellement de bienfaits non nécessaires ne nous dispense pas ce bienfait nécessaire à notre survie $^{18}$. Par conséquent, le législateur doit exister et doit être un homme. Il doit aussi posséder une caractéristique (khușusșiyya) que n'ont pas les autres hommes afin qu'on puisse le reconnaître ${ }^{19}$. Puis Avicenne affirme en guise de

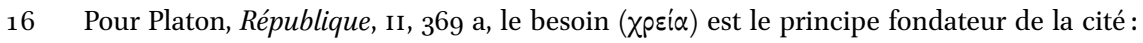
les hommes ne peuvent individuellement se suffire à eux-mêmes. Ils doivent s'associer pour échanger le produit de leur travail. La cité est donc en premier lieu un échange de services, une association d'intérêts entre les particuliers. Sur les traductions de Platon en arabe, cf. Gutas, "Platon 'Tradition arabe'. Plus spécifiquement sur les traductions arabes de la République, cf. Reisman, "Plato's Republic in Arabic".

17 Al-mu'āmalāt est un terme de figh désignant: "toutes les matières de droit au sens occidental du terme - statut personnel, droit commercial, droit pénal, etc. - et vise à mettre l'ensemble des relations humaines en harmonie avec les enseignements de la sharia", voir Chaumont, "Sharia" 829 .

18 Ce raisonnement est courant dans les discussions juridiques et théologiques du Xe siècle, notamment chez les Shāfi'ites. Sur ce point, cf. Shamsy, "The wisdom of God's Law". Shamsy observe: "The primary justification for the assumption that the sacred law was intended for the benefit of humankind appears to have been the divine attribute of wis$\operatorname{dom}(\text { hikma })^{\prime} 24$.

19 Voici la traduction en entier de ce passage de la Métaphysique du Shifä) : "Nous disons à présent qu'il est bien connu que l'homme se distingue du reste des animaux en ce qu'il ne peut avoir une vie convenable s'il s'isole seul en tant qu'individu, administrant ses affaires sans associé pour l'aider à [satisfaire] ses besoins fondamentaux. [Il est aussi bien connu] que [l'activité] de chaque homme doit être complémentaire (mukfiyyan) [de celle] d'un membre de son espèce ; [l'activité] de cet autre étant également complémentaire de celle du premier et [de celle] de l'un de ses semblables. Ainsi, l'un fournirait des légumes à l'autre et ce dernier fournirait du pain au premier ; celui-ci ferait de la couture pour l'autre 
conclusion : «Il est donc nécessaire qu'un prophète existe, et il est nécessaire que ce soit un homme ( fa-wājibun idhan an yūjada nabìwa wājibun an yakūna insānan) $»^{20}$.

Il nous faut comprendre pourquoi Avicenne conclut ce raisonnement en affirmant que l'existence du prophète est nécessaire. Pourquoi sans le prophète ne peut-il y avoir de sharía, entendue comme loi éthique et politique? Et pourquoi sans cette loi, ne peut-on envisager une authentique société humaine au sein de laquelle la justice est possible? Notons le lien étroit établit par Avicenne entre éthique et politique. Cette interconnexion est due au fait que, comme le notait Muhsin Mahdi, l'accent mis sur le devoir éthique de l'homme est le principe fondamental de la vie politique et sociale telle que la conçoivent aussi bien les philosophes que la Loi divine. La vie politique et la vie sociale se caractérisent par le devoir d'agir de façon vertueuse ${ }^{21}$.

Si seul le prophète est à même d'être le nomothète, c'est parce que la loi qui régit la cité est à la fois un code éthique et un code politique ou plutôt un code politique en tant qu'elle est un code éthique. Il nous faut essayer

et cet autre lui procurerait l'aiguille pour coudre, de sorte que lorsqu'ils se réuniraient leurs affaires seraient complémentaires. C'est la raison pour laquelle [les hommes] ont considéré comme nécessaire d'établir des cités et de former des associations. Quiconque parmi eux n'a pas fait attention dans la fondation de sa cité à la connaissance requise des règles nécessaires [pour fonder] une cité, et avec ses compagnons, s'est limité à [fonder] une simple assemblée serait d'un genre très dissemblable à celui des hommes et nierait leurs perfections. Quoi qu'il en soit cette sorte d'homme est obligée de former des assemblées et de chercher à ressembler aux citoyens des cités. Puisque cela est manifeste, il est nécessaire pour l'existence de l'homme et pour sa survie de s'associer. L'association ne s'achève qu'à travers la transaction réciproque (al-mu'āmala), de même qu'il est nécessaire pour [réussir cette association] d'user de toutes les autres causes dont [l'homme] dispose. Afin de pouvoir pratiquer des transactions, il est nécessaire de disposer de loi (sunna) et d'équité. Afin d'avoir la loi et l'équité, il est nécessaire d'avoir un législateur et un dispensateur de justice. Ce dernier, en tant qu'il s'adresse aux hommes et les incite à adhérer à la loi doit nécessairement être un homme. [Le législateur] ne peut pas laisser les hommes à leurs opinions concernant la loi, car alors ils divergent, chacun considérant comme juste ce que les autres lui doivent et injuste ce qu'il doit aux autres ( fa-yakhtalifün wa yarā kullun minhum mā lahu 'adlan wa mā 'alayhi zulman)", Shifä', Ilāhiyyāt, x, 2, 441. La dernière affirmation de ce passage de la Métaphysique du Shifä' est proche de celle qu'on trouve dans un pseudépigraphe arabe attribué à Platon, "L'ignorant (...) s'imagine que ce qui appartient à autrui est à lui", cf. Aflāțūn, Kitāb al-nawāmīs, 198. Georges Tamer a établi qu'Avicenne connaissait très probablement cet ouvrage, qui aurait été rédigé dans des milieux ismaéliens et soufis au x ${ }^{\text {ème }}$ siècle, cf. Tamer, "Politisches Denken".

20 Shifä, Ilāhiyyāt, $\mathrm{x}, 2,442$.

21 "The emphasis on man's duty is also the overarching principle of political and social life as seen by both the philosophers and the divine law. Political and social life are constituted by the duty to act in a virtuous way", Muhsin Mahdi, "The Political Orientation" 5 . 
de comprendre pourquoi seul le prophète est à même de révéler les lois qui servent de fondements éthiques et politiques à la cité.

Le prophète est un don de la providence divine ${ }^{22}$. Il apporte une loi, la sharīa, qui guide les hommes, règle leur comportement éthique et fonde le pacte politique. Ce sont les lois révélées par le prophète qui permettent aux hommes de ne pas laisser leurs corps dominer leurs âmes et l'empêchent de sombrer dans le mal moral. Le mal à l'égard des autres est donc d'abord et avant tout un mal envers soi-même; c'est un inversement des rapports de domination: les puissances concupiscibles et irascibles prennent le dessus et étouffent les élans de l'âme rationnelle. Un homme chez qui un tel inversement a lieu ne peut qu'être injuste envers ses concitoyens étant donné que seule la satisfaction de ses instincts lui importe. La justice est donc une vertu qui se cultive d'abord en soi-même en redonnant à ses puissances psychiques l'ordre de préséance qu'elles méritent ${ }^{23}$.

Cependant, alors même que les lois sont indispensables à la bonne marche de la cité, seul le recours à la providence divine peut leur conférer une réelle nécessité. En effet, les normes éthiques, selon Avicenne, ne sont pas accessibles à l'intellect humain par ses propres efforts contrairement aux lois physiques et mathématiques qu'il est en mesure de connaître en se tournant vers l'Intellect agent. Ainsi, des propositions telles «le tout est plus grand que la partie» sont universelles et peuvent être découvertes par l'intellect humain, qui va procéder par syllogisme et obtenir le moyen terme - de l'Intellect agent. Avicenne nous explique que ce mode de connaissance ne concerne pas les propositions éthiques telles que « la justice est bonne» ou «provoquer la douleur est mal». Les propositions de ce type font partie des opinions louables $\left(\bar{a} r \bar{a}^{\prime}\right.$ $m a \grave{h m u} \bar{u} d a)^{24}$. Ces propositions ne peuvent servir de prémisses aux syllogismes démonstratifs (qiyās burhānī); leur seul fondement est le fait d'être notoires $(l \bar{a}$

22 Sur la question de savoir comment la providence divine rend nécessaire l'existence du prophète, cf. Sebti, "Causalité secondaire". Le prophète est également présenté comme un don de la providence divine dans le traité pseudépigraphe arabe attribué à Platon (Aflāțūn), Kitāb al-nawāmīs.

23 Cette caractéristique permet de comprendre pourquoi la question du mal moral est abordée par Avicenne aussi bien dans les petits traités d'éthique de jeunesse (al-birrwa-l-ithm; al-akhlāq), dans ses commentaires coraniques (sūrat al-nās, sūrat al-falaq), que dans le dernier chapitre de la Métaphysique du Shifä', qui constitue l'exposé le plus complet de sa doctrine politique.

24 Dans son ouvrage intitulé Ibn Taymiyya's Theological Ethics Sophia Vasalou fait une analyse remarquable de la doctrine avicennienne de la nature des propositions éthiques. La présentation qui suit doit beaucoup à son travail, Ibn Taymiyya's Theological Ethics, 58 et ss. 
'umdata lahā illā al-shuhra $)^{25}$. Avicenne explique que la conscience morale, avec tous les jugements qu'elle implique, ne repose sur rien d'autre que sur des conventions sociales inculquées depuis le plus jeune âge aux hommes ${ }^{26}$. C'est

25 "Quant aux propositions notoires (al-mashhūrāt) qui relèvent de cet ensemble, certaines sont aussi ces propositions premières (al-awwaliyyāt), (...) et d'autres sont les points de vue appelés les points loués $(a l-m a h m m \bar{d} d a)$. A ces derniers, nous pourrions bien donner en propre le nom de propositions notoires (al-mashhüra), car elles n'ont d'autre pilier que la notoriété. Il s'agit de points de vue tels que, si quelqu'un s'isolait avec son intellect détaché du reste, son estimative et ses sens, s'il n'était pas éduqué à recevoir des propositions relatives à [ces points de vue] et à les reconnaître, si l'induction n'inclinait pas son opinion forte à un jugement à cause de la multiplicité des particuliers et si ce qu'il y a dans la nature de l'homme en fait de miséricorde, de honte, de pudeur, de sens de l'honneur, etc. n'appelait pas ces points de vue, l'homme ne se déciderait pas pour eux par soumission à son intellect, son estimative ou ses sens. Il en est ainsi, par exemple, lorsque nous jugeons que voler à un homme son bien est laid et que le mensonge est laid, tel qu'il ne faut pas s'y engager. De ce genre est l'appréciation qui se présente en premier à l'estimative de nombreuses personnes - quoique la loi religieuse ( $($ l-shar $)$ en détourne beaucoup de gens - sur la laideur de l'égorgement des animaux - et cela conformément à ce qu'il y a de tendresse (riqqa) dans l'instinct - aux yeux de celui qui a pareil instinct. Il s'agit de la plupart des gens. Or rien de tel n'est imposé nécessairement par l'intellect pur. Si l'homme se représentait lui-même dans son estimative comme créé tout d'un coup avec un intellect achevé, comme n'ayant rien écouté en fait d'éducation, comme n'ayant été soumis à aucune passion psychique ou à aucun trait de caractère, il ne déciderait rien à propos de pareilles propositions, bien plus, il lui serait possible de les ignorer et de suspendre [son jugement] à leur sujet. Il n'en est pas ainsi quand il décide que le tout est plus grand que la partie", Ishārāt, I 351-352. Ce passage a été traduit par Sophia Vasalou, Ibn Taymiyya's Theological Ethics, 59. Je reprends ici la traduction de Maroun Aouad dans "Les prémisses rhétoriques", 297-98.

26 A la fin de ce passage, il a recours à une démonstration problématique, semblable dans son articulation à la fameuse expérience de "l'homme volant". Avicenne affirme la possibilité pour l'homme de revenir à sa fitra, à sa nature originelle. En revenant à cette nature, l'homme prendrait alors conscience du fait que toutes les propositions éthiques ne sont rien d'autres que des conventions sociales dues à son éducation. L'argumentation avicennienne repose sur la possibilité de retrouver une nature humaine première, "nue" et pure de toute influence sociale ou culturelle (cet argument soulève de nombreux problèmes qui sont évoqués par S. Vasalou, Ibn Taymiyya's Theological Ethics, 6o et ss.). Sur la notion de fitra, voir Griffel, "Al-Ghazālīs Use of 'Original Human Disposition”, en particulier 11-32. F. Griffel note à juste titre que ce qui intéresse Avicenne n'est pas tant la question de la connaissance a priori que la connaissance qu'ont tous les êtres humains en commun lorsqu'ils n'ont que la perception sensible à leur disposition et sont dénués de toute influence sociale. Faut-il voir dans la conception de la fitra avicennienne une influence de la conception coranique de la fitra selon laquelle Dieu aurait créé l'homme selon une nature unique et immuable qu'aucune des vicissitudes de l'histoire ne saurait transformer? L'examen de cette question dépasserait le cadre de cet exposé. (Sur la notion de fitra dans le Coran, cf. 3o:30 : fitrata llāhi allatī fațara al-nās 'alayhā. Le Coran contient de nombreuses occurrences de la racine fṭr et une seule (celle sus-mentionnée) de fitra. La 
donc l'éducation qui inculque aux hommes dès l'enfance les normes éthiques leur permettant de vivre en société conformément à l'intérêt commun ${ }^{27}$.

Les propositions éthiques n'ont d'autres fondements que l'éducation et le conditionnement social. Cette caractérisation n'a pas pour effet de rendre ces propositions superflues. Nous avons vu, qu'Avicenne affirme clairement dans le chapitre 2 du livre x de la Métaphysique du Shifä', qu'il n'y a de vie possible pour l'homme que sociale, et que la vie en société ne peut se concevoir sans être encadrée par des normes éthiques. Sans ces normes, l'injustice la plus brutale régnerait et ruinerait tout projet de vie commune. Ainsi, Avicenne distingue deux points de vue; un point de vue épistémologique selon lequel les normes éthiques ne sont pas premières et sont le produit d'une expérience et d'un conditionnement social ; un point de vue proprement politique suivant lequel aucune vie citoyenne n'est concevable sans elles. Ce qui est important pour notre propos, c'est la doctrine selon laquelle ces normes éthiques - au vue de leur statut épistémologique - ne peuvent être déduites par la raison théorique. Si l'homme devait les déduire par sa raison pratique, ce serait par le biais d'une faculté psychique appelée par Avicenne « faculté estimative ».

Pour comprendre cela, il faut se remémorer quelques-uns des principes de la psychologie avicennienne. Lâme humaine a deux faces : l'une, son intellect théorique, est tournée vers le monde intelligible, l'autre, l'intellect pratique, est penchée vers le monde sensible. Ce qui relève du rapport de l'homme au monde sensible est du ressort de la faculté psychique «estimative». Cette faculté psychique ${ }^{28}$ est une faculté judicative qui intervient dans tous les jugements relatifs au sensible. Ainsi, elle intervient dans les jugements mettant en cause des émotions comme la colère, la peine, le désir, etc. Pour Avicenne c'est de cette faculté psychique que procède la plupart des activités animales ${ }^{29}$. Le jugement $(\mathrm{hukm})$ porté par l'estimative sur le sensible ne peut être identifié ni

notion de fitra occupe une place importante dans les débats des théologiens musulmans. Cf. Gobillot, La conception originelle.

27 "Lintérêt commun (mașlaha ) exige que parmi tous les actes qu'il peut accomplir, l'homme s'abstienne d'en accomplir certains. [Les hommes] apprennent cela lorsqu'ils sont jeunes et sont éduqués en ce sens. Ils s'habituent à entendre depuis le temps de leur enfance qu'ils ne doivent pas les accomplir, de sorte que cette croyance (itiqād) devient pour eux comme un instinct ( $k a-l$-ghariżì). Ces actes sont appelés "mauvais" (qabĭh), alors que les actes que l'on doit accomplir sont appelés 'bons' ( jamïla)", Shifä̀, Kitäb al-nafs, v, 1, 183.

28 Sur la doctrine des sens internes d'Avicenne, voir Sebti, Avicenne, 53-91.

29 "Lestimative est la puissance de jugement la plus importante chez les animaux; elle juge au moyen d'une impulsion qui provient de l'imagination, sans que ce jugement soit vérifié. C'est ce qui advient à l'homme qui éprouve une répulsion pour le miel en raison de sa ressemblance avec la bile. L'estimative juge qu'il en est ainsi et l'âme la suit même si l'intellect désapprouve. Les animaux, et les hommes qui leur sont semblables, ne suivent 
à l'affection sensorielle, ni au jugement intellectuel (al-tașdīq), qui porte sur les intelligibles. Ainsi, les jugements issus de la faculté estimative peuvent entrer en conflit avec ceux de l'intellect. Dans la partie de la logique de la Najāt correspondant aux Seconds Analytiques d'Aristote, Avicenne explique que parmi les jugements issus de l'estimative, certains sont vrais et d'autres faux ${ }^{30}$. Si l'homme devait fonder lui-même les normes éthiques, il dépendrait de cette puissance psychique dont les jugements sont aléatoires. C'est alors l'estimative qui lui ferait paraître «comme juste ce que les autres lui doivent et injuste ce qu'il doit aux autres $»^{31}$. La conséquence serait désastreuse car l'ordre social serait impossible à fonder. C'est la raison pour laquelle la providence divine intervient, pour assurer la possibilité de la vie citoyenne et donc la vie même de l'homme, puisqu'il n'y a pas de vie possible pour l'homme hors de la cité.

Pour ce qui est de sa vie éthique et politique, l'homme dépend de la révélation prophétique, qui seule peut lui enseigner comment fonder et maintenir une société juste: une société où chacun agit de manière vertueuse pour son bien propre (ici-bas et dans l'au-delà) ${ }^{32}$ et pour celui de la communauté.

dans l'accomplissement de leurs actions que ce jugement qui ne contient pas de discernement rationnel, mais résulte seulement d'une impulsion", Shifă', Kitāb al-nafs, IV, 3, 162. Comme exemple de jugement faux, Avicenne donne : "Le monde se termine dans le vide". En ce qui concerne les jugements vrais de l'estimative, c'est par exemple le fait que cette puissance ne peut se représenter deux corps dans un même lieu, l'intellect juge alors qu'un même corps ne peut être en même temps dans deux lieux différents. La force des jugements issus de l'estimative est telle qu'ils restent valides pour l'estimative, alors même qu'ils sont infirmés par l'intellect: "Ces jugements sont extrêmement puissants pour la pensée discursive. Seul l'intellect détruit certains de ces jugements qui, bien qu'ils soient détruits, demeurent dans l'estimative. C'est la raison pour laquelle ils ne se distinguent pas au commencement des prémisses rationnelles. Leur ressemblance est due au fait que la disposition naturelle (al-fitra) les certifie de la même manière qu'elle certifie les prémisses rationnelles", Najāt, 98-99. Ce passage est analysé par Hasnawi dans "La conscience de soi“, 287-89. Pour cette partie de la citation, j'ai repris la traduction de Hasnawi.

31 Shifä̀, Ilāhiyyāt, x, 2, 441.

32 C'est grâce à la religion et à ses cultes que les hommes peuvent surmonter leurs vils instincts et vivre ensemble en harmonie. Comme nous pouvons le voir dans le dixième livre d'Avicenne de la Métaphysique du Shifä', la Loi révélée par le prophète concerne les dogmes et les questions de dévotion, ainsi que les lois familiales, les transactions commerciales et les questions économiques (on retrouve la distinction bien connue des juristes musulmans entre mu'āmalāt et 'ibādāt). Le coran contient trois cent cinquante versets connus sous le nom de āyāt al-ahkām. Parmi eux, cent quarante concernent le dogme et les questions de dévotion, soixante-dix le mariage, le divorce et la paternité ; encore soixante-dix, les transactions commerciales (vente, prêt ...) et dix des questions d'économie, Shifä̀, Ilāhiyyāt, x, 2, 443-55. 
L'un et l'autre étant indissociables. Il n'y a donc pas de salut hors de la cité33. Cependant, alors que pour Fārābī, la cité est le lieu où l'homme réalise sa pleine perfection d'être rationnel, pour Avicenne, il ne s'agit que de la perfection morale de l'homme. Aussi, pour Avicenne, contrairement à ce que pensait son illustre prédécesseur, la fonction du philosophe et celle du prophète ne sont pas similaires. Le prophète apporte la sharî́a sans laquelle la vie sociale et politique est impossible. Il transmet aux hommes une connaissance inaccessible au philosophe. La fonction de nomothète et de princeps chez Avicenne n'est plus assignée au philosophe mais au prophète.

\section{$5 \quad$ Éthique et prophétie selon Averroès : une esquisse}

Averroès traite également à plusieurs reprises la question de la prophétie ${ }^{34}$. Dans son traité sur le raisonnement religieux, al-Kashf'an manāhij al-adilla $f i$ 'aqā’id al-milla ou L'explication des différentes sortes de preuves dans la doctrine religieuse, il mentionne souvent la prophétie comme fondement de la doctrine religieuse. Ce sujet est également abordé à plusieurs reprises dans son Tahäfut al-tahäfut ou Incohérence de l'Incohérence. La prophétie est également abordée dans son Traité décisif (Kitāb faṣl al-maqāl wa-taqrīr mā bayna al-sharīa wa-l-hikma min al-ittișāl).

Averroès rejette la possibilité d'une double vérité, l'une pour la religion et l'autre pour la philosophie et la sagesse (y compris la science). D'où sa célèbre sentence dans le Faṣl al-maqāl: « Car la vérité ne peut être contraire à la vérité, mais s'accorde avec elle et témoigne en sa faveur ${ }^{35}$.

Le philosophe cordouan considère que le discours du prophète agit à un autre niveau que celui du philosophe. Il ne s'adresse pas aux mêmes personnes que le discours philosophique. Averroès établit une division psychologique des êtres humains en trois groupes. Il y a ceux qui sont touchés par la rhétorique de la persuasion émotionnelle, à travers des récits qui affectent le cœur et l'imagination. D'autres donnent leur assentiment à un raisonnement dialectique fondé sur des postulats religieux, ce qui donne lieu à des interprétations

33 Cette dernière assertion est un écho à Fārābī selon lequel, point de salut hors de la cite vertueuse, cependant, il y a une difference de taille entre les deux philosophes que Miriam Galston résume parfaitement: "for Alfarabi, cities exist to make men good; for Avicenna, citizens are made good so that cities can exist", Galston, "Realism and Idealism" 570.

34 Pour un exposé de la doctrine de la prophétie d'Averroès, voir Taylor, "Averroes" 287-304.

35 Traité décisif, traduction M. Geoffroy, 118 (arabe); 119 (français). Sur cette question, cf. Taylor, "Truth does not contradict truth". 
conformes à la tradition religieuse. Le troisième groupe se compose de ceux qui sont des intellectuels bien informés et formés dans les arts philosophiques et qui utilisent le raisonnement de la logique et la méthode de la démonstration :

En effet, il existe une hiérarchie des natures humaine pour ce qui est de l'assentiment: certains hommes assentent par l'effet de la démonstration; d'autres assentent par l'effet des arguments dialectiques, d'un assentiment similaire à celui de l'homme de démonstration, car leurs natures ne les disposent pas à davantage; d'autres enfin assentent par l'effet des arguments rhétoriques, d'un assentiment similaire à celui que donne l'homme de démonstration aux arguments démonstratifs ${ }^{36}$.

Les deux premières donnent leur assentiment par des moyens qui peuvent en fait s'approcher de la vérité bien qu'il ne soit pas nécessaire qu'ils impliquent la vérité. La troisième, cependant, par la méthode même de la démonstration avec l'utilisation de prémisses connues pour être nécessaires et vraies et avec l'emploi d'une forme syllogistique valide peut atteindre la vérité en soi et nécessairement.

Ainsi, il n'y a qu'une seule vérité, mais elle est transmise de manière différente en fonction des capacités de chacun. Averroès insiste sur le fait que la révélation contient les trois niveaux de production de l'assentiment:

Et que la finalité de la Révélation n'est autre que d'enseigner tous les hommes, il fallait nécessairement quele Texte révélé comprît tous les types de méthodes de production de l'assentiment et de la représentation ${ }^{37}$.

Cela exige qu'en cas de désaccord sur des questions qui font l'objet d'études et d'enquêtes à la fois religieuses et philosophiques, la priorité soit donnée à l'interprétation philosophique et scientifique proprement dite. Pourtant, la majorité des gens ne sont pas capables de saisir cette distinction de discours et de concilier une telle interprétation avec le discours religieux, car ce dernier, de par sa nature même, se veut émotif et dialectiquement persuasif.

Bien sûr, Averroès affirme clairement que certains principes fondamentaux de la religion sont tels qu'ils doivent être acceptés par les gens des trois niveaux, à savoir l'existence de Dieu, son envoi de prophètes à l'humanité et une vie après la mort dans laquelle récompense et punition sont octroyées:

$36 \quad$ Traité décisif, 116 (arabe); 117 (français).

37 Traité décisif, 152 (arabe); 153 (français). 
La reconnaissance de l'existence de Dieu, des prophéties, de la béatitude et des tourments dans l'au-delà; car ces trois dogmes fondamentaux, les trois types d'arguments par l'effet desquels se produit immanquablement l'assentiment de tous les hommes à ce que la Loi les engage à connaître, les arguments rhétoriques, dialectiques et démonstratifs, aboutissent [également à en établir la véracité] ${ }^{38}$.

Averroès précise que certaines interprétations philosophiques peuvent semer le doute chez les gens ordinaires et les mener à une confusion dévastatrice. Pour cette raison, ces raisonnements ne peuvent être partagés en dehors du cercle du troisième groupe, celui des philosophes. Pour ceux qui sont incapables de comprendre à ce niveau le plus élevé, ces interprétations représenteraient une menace sérieuse pour les croyances religieuses, ce qui pourrait même mener à l'incrédulité. C'est le cas de la question de la nature et du sens de l'au-delà :

Et voilà la raison de notre opinion suivant laquelle l'interprétation pratiquée par des gens auxquels il est fait obligation de croire en le sens obvie, est infidélité : parce qu'elle conduit à l'infidélité. Quant aux hommes habilités à interpréter, et qui divulguent ces interprétations à l'intention de ces gens, ils les provoquent à l'infidélité. Or qui provoque à l'infidélité est un infidèle ${ }^{39}$.

La distinction du discours soutient donc la vie pratique des membres de la société, qui sont guidés par les enseignements religieux vers le bien dans leur vie d'individu ou de membre de communauté des croyants. Elle permet également que les enseignements religieux soient interprétés par ceux qui possèdent des compétences qualifiées, à condition qu'ils ne nuisent pas à ceux qui sont moins capables de comprendre :

Il nous faut savoir que la finalité de la Révélation se ramène à ceci : enseigner la science vraie et la pratique vraie (talìm al-ilm al-haqq wa-l-amal al-haqq). La science vraie, c'est la connaissance de Dieu - Béni et exalté soit-Il- et de l'ensemble des étants tels qu'ils sont - en particulier les plus sublimes d'entre eux -, et la connaissance de la béatitude et des tourments dans l'au-delà. La pratique vraie consiste dans l'accomplissement

$3^{8} \quad$ Traité décisif, 138 (arabe); 139 (français).

39 Traité décisif, 144 (arabe) ; 145 (français). 
des actes qui assurent la béatitude, et l'évitement des actes qui valent les tourments. La connaissance de ces actes se nomme la science pratique ${ }^{40}$.

Dans le Faṣl al-maqāl, la nécessité de la prophétie est affirmée à maintes reprises tout au long du texte, de même que la division des niveaux de signification qui convient aux trois groupes de personnes que j'ai mentionnés. Averroès affirme que les trois catégories ont la capacité de connaître (al-márifa) de manière affirmative l'existence de Dieu, son envoi de prophètes à l'humanité et la récompense et le châtiment dans l'au-delà. Telles sont les croyances fondamentales qui conduisent les êtres humains à l'action appropriée et toutes sont affirmées dans le Coran. Ainsi, éthique et politique sont intrinsèquement liées. Une société juste est une société qui donne à chacun, selon ses capacités intellectuelles, la capacité d'agir dans le sens de la justice. Il n'y a pas de politique sans éthique et la loi révélée, la sharîa permet la réalisation de ces deux niveaux.

Dans le Tahāfut al-tahāfut, l'affirmation de la prophétie est supposée tout au long du texte. Averroès y affirme que les miracles sont des principes de la religion qui ne doivent pas être remis en question ou mis en doute parce qu'ils sont au-delà de toute appréhension humaine. Leur valeur réside dans le fait de guider les êtres humains vers la vertu : ils ont donc une fonction centrale dans l'établissement de la science pratique. Néanmoins, les miracles accomplis par les prophètes ne doivent pas être considérés comme la réalisation de ce qui est logiquement impossible, mais peut-être plutôt ce qui est possible en soi mais pas possible pour les êtres humains. Cependant, le plus sûr de tous les miracles est le Coran lui-même selon Averroès, qui par cette affirmation demeure fidèle à l'orthodoxie musulmane ${ }^{41}$ :

Le plus clair des miracles est le Vénérable Livre d'Allah, dont l'existence n'est pas une interruption du cours de la nature assumée par la tradition, comme la transformation d'un bâton en serpent, mais sa nature miraculeuse est établie par la perception et la considération de chaque homme qui a été ou sera jusqu'au jour de la résurrection. Ce miracle est donc de loin supérieur à tous les autres ${ }^{42}$.

De plus, la vraie réalité de la nature du prophète en tant que prophète se trouve "dans l'acte de faire connaître le mystérieux (al-ilām bi-l-ghuyūb) et d'établir

$40 \quad$ Traité décisif, 150 (arabe); 151 (français).

41 Sur le dogme de l'inimitabilité du Coran, cf. Urvoy, "Inimitabilité du Coran" 419-20.

42 Averroès, Tahāfut al-tahāfut, 515-16. 
des lois (wad' al-sharāं $\left.i^{\prime}\right)$ qui sont conformes à la vérité et qui produisent des actes qui détermineront le bonheur de l'humanité entière"43. La véritable affirmation du prophète réside en cela et non dans le fait d'accéder à des connaissances cachées au moyen de rêves, connaissances qui peuvent avoir des explications naturelles ${ }^{44}$.

Les lois religieuses qui proviennent de Dieu, par les prophètes, ainsi que de la raison humaine naturelle sont nécessaires pour la construction de la bonne structure politique et sociale. Les principes de base communs à toutes les religions reçus des prophètes et des législateurs tirent leur valeur du fait qu'ils orientent les êtres humains pour les éloigner du vice et les diriger vers des actions vertueuses ${ }^{45}$.

Dans le Kashfl'affirmation de la nécessité du prophète repose sur deux principes: l'existence évidente des prophètes en tant que porteurs des lois religieuses à travers la révélation, qui permet de faire connaitre les actions justes pour atteindre le bonheur, et la fonction évidente des prophètes en tant que dépositaires des lois religieuses dans la révélation de Dieu ${ }^{46}$. Il n'est pas nécessairement vrai que tout faiseur de miracles soit un prophète comme le pensent les théologiens, mais il est vrai que le Coran lui-même avec sa connaissance des lois religieuses, du bon comportement humain et de la nature de Dieu est considéré comme miraculeux pour ses conséquences. En cela, la preuve du prophète de Dieu est le bienfait inestimable du Coran pour guider les êtres humains, tout comme la preuve de l'utilité du médecin réside dans la guérison réelle des malades. ${ }^{47}$

L'existence et la nature de la prophétie sont considérées comme évidentes dans l'expérience des êtres humains en relation avec le message du Coran, qui fournit des lois religieuses pour guider l'humanité vers le bien.

Dans son commentaire sur la Métaphysique d'Aristote, Averroès affirme explicitement que la forme la plus parfaite de culte de la Divinité se trouve dans

43 Averroes, Tahäfut al-tahäfut, ${ }^{16}$.

44 Averroes, Tahäfut al-tahäfut, 533 .

45 "La catégorie qui est appelée "messagers" et "prophètes" est connue par elle-même. Cette catégorie d'homme est celle qui établit les lois (al-sharäà) pour les hommes par une révélation venant de Dieu et non par un enseignement humain (...) Il y a là des individus parmi les hommes à qui une révélation est donnée par laquelle ils transmettent aux hommes des choses concernant des connaissances et des actes vertueux grâce auxquels est achevée leur béatitude ; ils leur interdisent des croyances mauvaises et des actes vils. Telle est l'action des prophètes", Averroes, Kashf, 215.

46 Averroes, Kashf, 213.

47 Averroes, Kashf, 202. 
la connaissance de Dieu et des créatures dans la science de la métaphysique. Cette connaissance est une obligation religieuse spécifique aux philosophes (al-sharīa al-khāsșa bi-l-hukamä). Il semble donc raisonnable de conclure qu'Averroès considère la philosophie et ses sciences comme étant le contenu de vérité le plus complet et le plus précis; le plus haut niveau de connaissance et de compréhension accessible aux hommes. Dans cette perspective, la religion - qui est indispensable au développement politique humain ${ }^{48}$ - est comme une science pratique aristotélicienne en ce qu'elle concerne la bonne et juste conduite dans la réalisation d'un but que l'on atteint par l'action, et ne concerne pas la connaissance en soi de la vérité. La révélation transmet à tous les hommes, philosophes comme hommes ordinaires, la connaissance des trois vérités que j'ai évoquées (reconnaissance de l'existence de Dieu, des prophéties, de la béatitude et des tourments dans l'au-delà) - connaissances qui de toutes façons seraient accessibles aux philosophes; cependant, elle est indispensable dans sa fonction éthique et politique dans la mesure où sans elle, les hommes du commun ne pourraient avoir de normes pour réguler leurs actions et vivre avec leur semblables dans l'harmonie.

Je ne peux, dans le cadre de cet article, entrer dans les détails de ce qui différencie la prophétologie d'Avicenne de celle d'Averroès. Notons toutefois que pour Avicenne, le contenu du message prophétique n'est pas accessible autrement que par la révélation et que, de ce fait, le prophète est supérieur au philosophe dans la mesure où, outre la connaissance noétique qu'il partage avec ce dernier, il est porteur d'une autre connaissance - émanant des âmes célestes et reçue par son imagination. La valeur éthique (et donc politique) du message prophétique pour Avicenne ne tient donc pas au fait que le prophète rende accessible aux moyens de symboles et d'images une vérité métaphysique qui ne serait accessible qu'aux philosophes, mais au fait que le prophète est le seul homme à bénéficier de cette connaissance du monde invisible ('âlam $a l$-ghayb), et qu'il est celui à qui la Loi est révélée. En ceci, la thèse d'Avicenne est radicalement distincte de celle d'Averroès. Néanmoins, on retrouve chez les deux philosophes, ainsi que nous l'avons vu, l'affirmation de la fonction éthique et politique du prophète. Bien qu'à l'instar des autres falāsifa Avicenne rende compte rationnellement de la fonction du prophète, notamment des modalités épistémologiques de réception de la révélation, le fait qu'il considère que le prophète est supérieur au philosophe - lequel ne peut se substituer au premier en tant que nomothète - confère à sa prophétologie un statut à part. 


\section{Bibliographie}

\section{Sources}

Aflāțūn. Kitāb al-nawāmīs li-Aflāṭūn, in 'A.-R. Badawī, ed., Aflāṭūn fì l-islām, Beirut, Dār al-Andalus, 1982, 197-234.

Averroès, see Ibn Rushd.

Avicenne, see Ibn Sīnā.

Fārābī. Kitāa Taḥ̣̂̄l al-sacāda, ed. 'Ali Bū Mulḥam, Beirut, Dār wa Maktabat al-Hilāl, 1995 .

Ibn Rushd. Traité décisif (Faṣl al-maqāl), traduction M. Geoffroy, Paris, GF, 1996.

Ibn Rushd. Kashf manāhij al-adilla fì 'aqā'id al-milla, éd. M. Qāsim, zème édition, Maktabat al-Anglū al-mișriyya, Cairo, 1964.

Ibn Rushd. Tahāfut at-tahāfut, éd. M. Bouyges, Beirut, Dār al-Mashriq, 1992.

Ibn Sīnā. Al-Shifā’’ Al-Ilāhiyyāt, ed. G. C. Anawati et alii., Cairo, al-Hay’a al-āmma li-shu'ūn al-mațābi' al-Amīriyya, , 1959.

Ibn Sīnā. Al-Shifā', Kitāb al-nafs, ed. G.C. Anawati et alii., Cairo, al-Hay’a al-āmma li-shu'ūn al-Mațābi' al-Amīriyya, 1975.

Ibn Sīnā. Kitāb al-Najāt, ed. M. Fakhri, Manshūrāt Dār al-āfāq al-jadīda, Beirut, 1985, 98-99.

Kindī. Rasā'il al-Kindī al-falsafiyyya, ed. Abū Rīdā, Cairo, Dār al-Fikr, 1950, 363-384, 373 ; English translation: P. Adamson and P. Pormann, The Philosophical Works of al-Kindī, Oxford, Oxford University Press, 2012.

Rāzī, Abū Ḥātim. Kitāb alāam al-nubuwwwa, ed. Ṣ. al-Sāwī, Teheran, Anjuman-i Shāhinshāhī-yi Falsafa-yi Īrān, 1977.

Shahrastānī. Kitāb al-milal wa-l-niḥal, ed. A. F. Muḥammad, 3 vols, Beirut, Dār al-kutub al-ilmiyya, 1992.

Shahrastānī. Le livre des religions et des sectes, vol. II. Traduction avec introduction et notes par J. Jolivet et G. Monnot, Paris, Peeters, Unesco 1993.

\section{Études}

Adamson, P. Al-Kindi (Great Medieval Thinker), Oxford, Oxford University Press, 2007.

Adamson, P. and Pormann, P. The Philosophical Works of al-Kindī, Oxford, Oxford University Press, 2012.

Aouad, M. “Les prémisses rhétoriques selon les Ǐsāāāt d'Avicenne”, in Ph. Büttgen et alii, eds. Théories de la phrase et de la proposition de Platon à Averroès, Paris, PUF, 1999, 281-304.

Brague, R. "Note sur la traduction arabe de la Politique, derechef, qu'elle n'existe pas", in P. Aubenque, ed. Aristote politique, Paris, PUF, 1993, 423-433. 
Hasnawi A. "La conscience de soi chez Avicenne et Descartes", in J. Biard and R. Rashed, eds., Descartes et le Moyen Age, Paris, Vrin, 1997, 287-289.

Galston, M. "Realism and Idealism in Avicenna's Political Philosophy", The Review of Politics, 41, 4 (Oct. 1979), 561-577.

Gobillot, G. La conception originelle. La fitra, ses interprétations et fonctions chez les penseurs musulmans, Cairo, Institut français d'archéologie orientale, 2000.

Griffel, F. al-Ghazālī's Philosophical Theology, Oxford, Oxford University Press, 2009.

Griffel, F. “Al-Ghazālì's Use of 'Original Human Disposition' ( fițra) and its Background in the Teachings of al-Fārābī and Avicenna", The Muslim World 102 (2012), 1-32.

Gutas, D. Greek Thought, Arabic Culture: The Graeco-Arabic Translation Movement in Baghdad and Early 'Abbāsid Society (2nd-4th/8th-1oth centuries), Routledge, London-New-York, 1998.

Gutas, D. "Platon. 'Tradition arabe", in Dictionnaire des Philosophes antiques, 7 vols., Paris, CNRS, Éditions, 2012., Vol. v (Paccius à Plotin), 845-863.

Gutas, D. "The meaning of madanī in al-Fārābī's 'Political' Philosophy", Mélanges de l'Université Saint Joseph LVII (2004), 259-282.

Janssens, J. "Ibn Bājja and Aristotle's Political Thought", in V. Syros, ed. Well Begun is Only Half Done: Tracing Aristotle's Political Ideas in Medieval Arabic, Syriac, Byzantine, and Jewish Sources (Medieval and Renaissance Texts and Studies, 338), Tempe, Arizona, ACMrs, 2011, 73-95.

Mahdi, M. "The Political Orientation of Islamic Philosophy", Occasional papers series Georgetown University, Georgetown University, Center for Contemporary Arab Studies, 1982, 1-15.

Rashed, M. “Abū Bakr Rāzī et la prophétie", MIDEO 27 (2008), 169-182.

Reisman, D. "Plato's Republic in Arabic a newly discovered passage", Arabic Sciences and Philosophy 14 (2004), 263-300.

Sebti, M. Avicenne. L'âme humaine, Paris, PUf, 2000.

Sebti, M. "Causalité secondaire et providence divine chez Avicenne", Ishraq: Islamic Philosophy Yearbook, vol. 9 (2019), Moscow, Sadra Publishers, 233-256.

Shamsy, A. "The Wisdom of God's Law : Two Theories", in R. Peters and R. Gleave,, eds., Islamic Law in Theory. Studies in Jurisprudence in Honor of Bernhard Weiss, Leiden, Brill, 2014, 9-37.

Shihadeh, A. The Theological Ethics of Fakhr al-Dīn al-Rāzī, Leiden, Brill,20o6.

Tamer, G. "Politisches Denken in pseudoplatonischen Arabischen Schriften", Mélanges de l'Université de Saint-Joseph 75 (2004), 303-335.

Taylor, R. "Averroes and the Philosophical Account of Prophecy", Studia Graeco-Arabica 8 (2018), 287-304.

Taylor, R. “Truth does not contradict truth' : Averroes and the Unity of Truth", Topoi 19 (2000), 3-16. 
Urvoy, M.-T. “Inimitabilité du Coran”, in Amir-Moezzi, ed. Dictionnaire du Coran, Paris, Robert Laffont, 2007, 419-420.

Vasalou, S. Ibn Taymiyya's Theological Ethics, Oxford, Oxford University Press, 2016.

Vasileos, S. "A Note on the Transmission of Aristotle's Political Ideas in Medieval Persia and Early-Modern India. Was there Any Arabic or Persian Translation of the Politics ?", Bulletin de philosophie médiévale 5o (2008), 303-309. 